\title{
Behavioral insomnia in infants and young children
}

\author{
Eun Kyeong Kang, MD, PhD', Seung Soo Kim, MD, PhD² \\ ${ }^{1}$ Department of Pediatrics, Dongguk University Ilsan Hospital, Goyang, Korea; '2Department of Pediatrics, Soonchunhyang University Cheonan Hospital, Cheonan, \\ Korea
}

In infants and young children, bedtime problems and night waking are common and the main presentations of insomnia. Poor sleep may critically impact the daytime functioning and mood of the child and their caregivers. A comprehensive sleep history, a sleep diary/log, and the BEARS (Bedtime problems, Excessive daytime sleepiness, Awakenings during the night, Regularity and duration of sleep, and Sleep-disordered breathing) sleep screen are useful for diagnosing sleep problems in young children. Behavioral therapies for this type of insomnia include extinction, bedtime fading with positive routines, and scheduled awakening. Previous studies of behavioral interventions for young children showed significant improvements in sleep-onset latency, night waking frequency, and night waking duration. Parent education about their child's sleep, bedtime routines, and sleep hygiene is essential for treatment.

Key words: Sleep, Insomnia, Pediatric, Behavioral interven tion

\section{Key message}

- Behavioral insomnia is common and affects a child's daytime functioning and emotions.

- Diagnosing insomnia using the child's sleep history, a sleep diary/log, and sleep questionnaires is important.

- Behavioral intervention, the main treatment, involves creating positive associations with sleep, establishing a consistent sleep schedule and bedtime routines, and the development of selfsoothing skills.

\section{Introduction}

Nocturnal wakefulness is normal during early infancy and usually decreases substantially over the first few months of life. Thus, the majority of children sleep through the night by 6 months of age. ${ }^{1)}$ Differentiating sleep problems from normal physiologic changes in development can be challenging for clinicians. Sleep problems are commonly referred to as "bedtime problems and night waking" in infants and young children and insomnia in older children and adolescents. ${ }^{2)}$

Insomnia affects both sleep duration and quality. Total mean sleep duration is 14.2 hours (standard deviation [SD], 1.9 hours) at 6 months of age versus a mean 8.1 hours (SD, 0.8 hours) at 16 years of age. ${ }^{3)}$ The American Academy of Sleep Medicine recommends the amount of sleep per 24 hours for pediatric populations to promote optimal health ${ }^{4)}$ (Table 1). Chronic insufficient sleep quantity and inadequate sleep quality can impact a child's daytime functioning, causing daytime behavior problems, cognitive impairment, and mood disturbances. ${ }^{5)}$ Sleep problems in young children disrupt their parents' sleep, affecting their mood and daytime functioning as well. 6,7)

Chronic health problems (e.g., asthma, epilepsy, or atopic dermatitis) and developmental disorders will also adversely affect sleep. ${ }^{8)}$ This review describes behavioral insomnia of infants and young children and details its common interventions.

\section{Definition and subtypes of childhood insomnia}

Considering its characteristic features, the International Classification of Sleep Disorders (ICSD) 2nd edition classified pediatric insomnia independently as "behavioral insomnia of childhood (BIC)". ${ }^{9)}$ In the ICSD 3rd edition, pediatric insomnia was integrated into "chronic insomnia.",10) Considering the distinctive characteristics of pediatric insomnia, the text mentions three subtypes of pediatric insomnia: (1) sleep-onset association type, a "child's dependency on specific stimulation, objects, or setting for initiating sleep or returning sleep following

Table 1. Sleep amounts recommended by the American Academy of Sleep Medicine for pediatric populations

\begin{tabular}{lc}
\hline Age & Recommended total sleep time (hr) \\
\hline 4-12 Months & $12-16$ \\
$1-2$ Years & $11-14$ \\
$3-5$ Years & $10-13$ \\
$6-12$ Years & $9-12$ \\
$13-18$ Years & $8-10$ \\
\hline
\end{tabular}


an awakening; in the absence of these conditions, sleep-onset is significantly delayed;" (2) limit-setting type, "bedtime stalling or bedtime refusal that is met with and reinforced by inadequate limit-setting by a caregiver;" and (3) mixed type, "sleep-onset association difficulties and bedtime resistance."10)

Sleep associations are certain behaviors or environments that customarily appear at the time of sleep-onset that the child learns to need to fall asleep. ${ }^{5)}$ In the presence of a negative or inappropriate sleep-onset association, parental interventions (e.g., rocking, nursing, swinging) are required for the child to initiate sleep or return to sleep after a night awakening., ${ }^{59-11)}$ Therefore, frequent night awakenings are the presenting symptom of the sleep-onset association type. ${ }^{9,11)}$

The BIC limit-setting type, which usually begins after 2 years of age, occurs when the child refuses to go to bed. ${ }^{5,10)}$ Bedtime refusal and delaying tactics include attempts to delay bedtime (e.g., watching additional television) and the bedtime routine (e.g., requesting another story) or "curtain calls" after lights out (e.g., needing another story, hug, drink). ${ }^{5,11)}$ It occurs when there are few or no limits instituted by parents around sleep behaviors (e.g., allowing the child to fall asleep while watching television or to play games until lights out). ${ }^{11)}$ Parents may also institute limits in an inconsistent way (e.g., allowing the child to stay up late on weekends). ${ }^{5,111}$

Using a latent class analysis, Bruni et al. ${ }^{12)}$ recently suggested three pediatric insomnia subtypes and advocated different therapeutic approaches for each as follows: (1) presence of restless legs syndrome in dopaminergic dysfunction; (2) frequent depression and/or mood disorders in serotonergic dysfunction; and (3) common allergies and/or food intolerance in histaminergic dysfunction.

\section{Prevalence}

The prevalence of pediatric insomnia is reportedly $5 \%-20 \%$, affecting up to $75 \%$ of children with neurodevelopmental comorbidities. ${ }^{13-15)}$ In Korea, a study of patients visiting secondary and primary pediatric outpatient clinics reported a 13.2\% prevalence of pediatric insomnia in children and adolescents: $21 \%$ at age $0-1$ year, $13 \%$ at age $2-5$ years, $7 \%$ at age $6-12$ years, and $6 \%$ at age $13-18$ years. ${ }^{16}$ Similar to the prevalence of general pediatric insomnia, bedtime problems and frequent night waking occur in 20\%-30\% of infants and young children. ${ }^{17)}$

The prevalence of sleep problems including insomnia may vary depending on cultural and regional factors. ${ }^{18,19)}$ Children from predominantly Asian (P-A) countries (China, Hong Kong, India, Japan, Korea, Malaysia, Philippines, Singapore, Thailand) had significantly increased parental perception of sleep problems than those from predominantly Caucasian (P-C) countries (Australia-New Zealand, Canada, United Kingdom, United States) (Table 2). ${ }^{18)}$

Co-sleeping, broadly defined as room-sharing and bed-sharing, has generated a considerable amount of controversy. It is considered a factor that increases the chronic nature of sleep problems in P-C countries. ${ }^{11)}$ Co-sleeping is common in P-A countries, with no change across the preschool years. ${ }^{11,18,19)}$ However, when analyses of variance covarying with cosleeping were calculated that compared the predictors of sleep problems (night waking, sleep-onset latency, bedtime, nighttime sleep duration) between P-C and P-A countries, a significant difference was seen only in sleep-onset latency. ${ }^{18)}$

Table 2. Prevalence of sleep problems in infants and young children.

\begin{tabular}{|c|c|c|c|c|c|c|}
\hline Study & Country & $\begin{array}{c}\text { Prevalence } \\
\text { (\%) }\end{array}$ & $\begin{array}{l}\text { Sample size } \\
\text { (n) }\end{array}$ & $\begin{array}{l}\text { Age } \\
\text { (yr) }\end{array}$ & Case definition & Questionnaire \\
\hline Richman et al. ${ }^{41)}$ (1975) & UK & 12.5 & 705 & 3 & Sleep problem & $\mathrm{BSQ}^{42)}$ \\
\hline Camhi et al. ${ }^{43)}(2000)$ & US & 15.9 & 138 & $3-6$ & DIMS & Author defined \\
\hline Archbold et al. ${ }^{44)}$ (2002) & US & 20.6 & 399 & $2.0-4.9$ & Insomnia & $P S Q^{45)}$ \\
\hline Jiang et al. ${ }^{46)}(2007)$ & $\mathrm{CN}$ & 66.0 & 764 & $0.5-1.9$ & Sleep problem & Author defined \\
\hline Mindell et al. ${ }^{47)}(2009)$ & US & 9.3 & 836 & $0-6$ & Sleep problem & Author defined \\
\hline Zhang et al. ${ }^{48)}(2009)$ & HK & 3.3 & 793 & 6 & Insomnia & Author defined \\
\hline \multirow[t]{3}{*}{ Mindell et al. ${ }^{49)}(2010)$} & P-C: AU, CA, NZ, UK, US & 26.3 & 7,960 & $0-3$ & Sleep problem & $\mathrm{BISQ}^{21)}$ \\
\hline & P-A: CN, HK, IN, ID, KR, JP, MY, PH, SG, TW, TH, VN & 51.9 & 21,327 & & & \\
\hline & $\mathrm{KR}$ & 46.9 & 1,036 & & & \\
\hline Simola et al. ${ }^{50)}(2012)$ & Finland & 12.1 & 481 & $3-6$ & DIMS & $\operatorname{SDSC}^{51)}$ \\
\hline \multirow[t]{3}{*}{ Mindell et al. ${ }^{18)}(2013)$} & P-C: AU-NZ, CA, UK, US & 18.4 & 1,139 & $3-6$ & Sleep problem & $\mathrm{BCSQ}^{21)}$ \\
\hline & P-A: CN, HK, ID, KR, JP, MY, PH, SG, TH & 24.2 & 1,447 & & & \\
\hline & $\mathrm{KR}$ & 15.1 & 312 & & & \\
\hline Kim et al. ${ }^{16)}(2017)$ & KR & 15.2 & 678 & $0-5$ & Insomnia & $P S Q^{45)}$ \\
\hline
\end{tabular}

DIMS, disorders initiating and maintaining sleep; BSQ, behavioral screening questionnaire; $P S Q$, pediatric sleep questionnaire; BISQ, brief infant sleep questionnaire; SDSC, sleep disturbance scale for children; BCSQ, brief child sleep questionnaire.

Countries: AU, Australia; CA, Canada; CN, China; HK, Hong Kong; ID, India; IN, Indonesia; JP, Japan; KR, South Korea; MY, Malaysia; NZ, New Zealand; P-A, predominantly Asian; P-C, predominantly Caucasian; PH, Philippines; SG, Singapore; TH, Thailand; TW, Taiwan; VN, Vietnam. 


\section{Evaluation}

\section{Comprehensive sleep history}

The child's documented sleep history should include a detailed assessment of the sleep schedule on weekdays and weekends, the child's sleep environment (e.g., lighting, noise level, roomsharing with parents or siblings, bed type), sleep habits (e.g., parental involvement at bedtime and at awakening, frequency of night feeding), and bedtime routines. ${ }^{5)}$

\section{Sleep diary/log}

When a child's sleep history is unclear, the use of sleep diaries or sleep logs can be helpful in providing a more accurate overview of sleep patterns. ${ }^{5)}$ Asking parents to keep a diary of their child's sleep for at least a week is needed. Sleep diary information includes bedtime, time the child falls asleep, time and duration of night waking, morning waking time, and daytime nap times and durations. ${ }^{8}$ Parents can use a sleep diary application for smartphones (e.g., Kids Sleep Dr) to capture the data. A sample sleep $\log$ that was written for a 13-month-old baby with behavioral insomnia is shown in Fig. 1.

\section{BEARS sleep screen and sleep questionnaire}

The BEARS (Bedtime problems, Excessive daytime sleepiness, Awakenings during the night, Regularity and duration of sleep, and Sleep-disordered breathing) sleep screen is a useful tool that can be easily implemented during the well-child examination across the pediatric age spectrum. ${ }^{5,20)}$ (Table 3) The Brief Infant Sleep Questionnaire (BISQ), a sleep questionnaire for infants/ toddlers (0-29 months), ${ }^{21)}$ consists of 13 items with three main categories (nocturnal sleep duration, night waking, and method of falling asleep). There is a Korean version of the BISQ (Appen$\operatorname{dix} 1)$.

\section{Objective assessments: actigraphy and videosomnography}

Actigraphy is a popular sleep assessment tool that detects movement using a watch-like monitor positioned on the wrist or ankle. However, it carries the issue of interdevice reliability and a high false positivity for scoring wakefulness in infants. ${ }^{22} \mathrm{~A}$ recent longitudinal study of infant nocturnal wakefulness showed weak agreement rates between actigraphy and parental reports (sleep diaries and BISQ). ${ }^{23)}$

Videosomnography captures the infant's overnight sleep using time-lapse recording. Despite its accuracy, it is expensive and labor-intensive.

\section{Treatment}

\section{Behavioral interventions}

A recent meta-analysis and studies of behavioral interventions for pediatric insomnia concluded that behavioral treatments for young children result in significant improvements in sleep-onset latency, night waking frequency, and night waking duration. 2,17,24,25) The goals of most behavioral interventions include some combination of creating positive associations with sleep, establishing a consistent sleep schedule and bedtime routine, and the development of relaxation or self-soothing skills. ${ }^{26)}$ (Table 4) Although behavioral interventions for pediatric insomnia are beneficial, they must be personalized according to the child's

Table 3. BEARS sleep screen

\begin{tabular}{lc} 
Sleep domain & $\begin{array}{c}\text { Trigger questions for young children } \\
\text { (2-5 years) }\end{array}$ \\
\hline Bedtime problems & $\begin{array}{c}\text { Does your child have any problems } \\
\text { going to bed or falling asleep? }\end{array}$ \\
Excessive daytime sleepiness & $\begin{array}{c}\text { Does your child seem overly tired or } \\
\text { sleepy during the day? } \\
\text { Does your child still take naps? }\end{array}$ \\
Awakenings during the night & $\begin{array}{c}\text { Does your child frequently awaken at } \\
\text { night? }\end{array}$ \\
Regularity and duration of sleep & $\begin{array}{c}\text { Does your child have a regular bedtime } \\
\text { and wake time? }\end{array}$ \\
Sleep-disordered breathing & $\begin{array}{c}\text { Does your child snore or have difficulty } \\
\text { breathing at night? }\end{array}$ \\
\hline
\end{tabular}

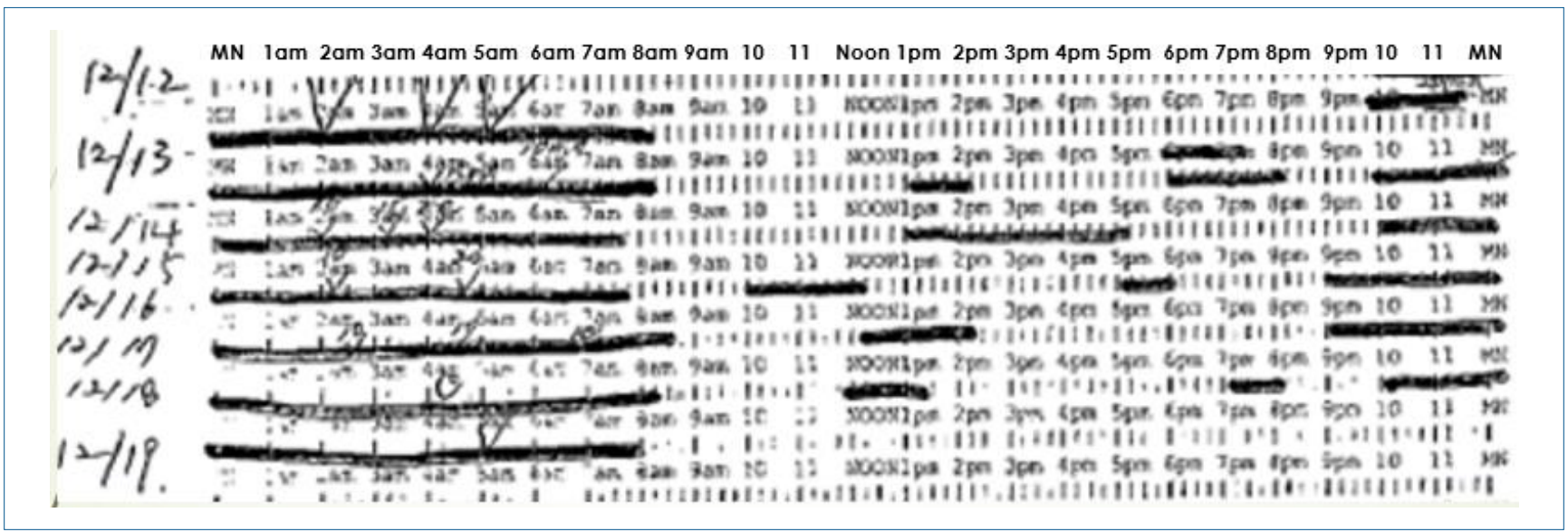

Fig. 1. This is a sample sleep log that was recorded for a 13-month-old baby with behavioral insomnia. The thick lines indicate the time of sleep, and check mark shows the time when the baby awakened during sleep. Note the irregular napping from 10 AM to 8 PM. (Courtesy of Professor Kyu Young Chae/CHA Bundang Medical Center, CHA University) 
Table 4. Summary of interventions for behavioral insomnia

\begin{tabular}{|c|c|c|c|}
\hline Behavioral intervention & Useful conditions & Characteristics & Cues for success \\
\hline Unmodified extinction & Sleep-onset BIC, for infants & $\begin{array}{l}\text { Faster effect } \\
\text { Distressing to parents }\end{array}$ & Consistent ignoring of crying \\
\hline Graduated extinction & Sleep-onset BIC, for infants & Less distressing than unmodified extinction & Consistent ignoring of crying \\
\hline Positive routines/faded bedtime & $\begin{array}{l}\text { Limit-setting BIC, mainly for } \\
\text { school-aged child }\end{array}$ & Optimally, should avoid daytime nap & Successful bedtime routines \\
\hline Scheduled awakenings & Frequent night waking, for infants & Increase sleep consolidation & Wake at relatively fixed hours \\
\hline
\end{tabular}

BIC, behavioral insomnia of childhood.

separation anxiety. ${ }^{27)}$

\section{1) Extinction}

The primary goal for the child is to learn to self-soothe. A study from 1988 showed that systematic ignoring (extinction) produced faster improvement than scheduled awakening and was equally effective at reducing nocturnal awakening and cry. ing in infants and young children. ${ }^{28)}$ Unmodified extinction (the "cry it out" approach) procedures involve having the parents put the child to bed and then ignoring the child's crying until the next morning except to check for their physical wellbeing. ${ }^{17)}$ In this approach, parents should consistently ignore the child's cries. Many studies have reported that this method is effective at treating sleep settling and night waking. ${ }^{2,29}$ However, unmodified extinction procedures are stressful for some parents. Disturbing neighbors with the noise are a practical concern in densely occupied buildings, ${ }^{30)}$ like those in Korea. Thus, "extinction with parental presence" or "gradual extinction" is more acceptable in such situations. ${ }^{17}$

\section{2) Graduated extinction}

Graduated extinction techniques gradually limit parental intervention in sleep initiation. Parents are instructed to ignore bedtime crying for specified periods by employing a fixed schedule (e.g., every 5 minutes) or waiting for progressively long. er intervals (e.g., 5 minutes, then 10 minutes, then 15 minutes) before checking on their child. ${ }^{17,31)}$ The parents should minimize interactions during brief check-ins ( 15 seconds to 1 minute). The efficacy of such methods has been shown in clinical studies. ${ }^{2,17)}$ A recent randomized controlled trial of behavioral interventions for infant sleep problems demonstrated large decreases in sleep latency for the graduated extinction and bedtime fading groups and significant decreases in awakenings for the graduated extinction group. ${ }^{25)}$

3) Positive routines and faded bedtime with response cost

Bedtime fading ${ }^{32)}$ is helpful when the child's sleep-onset is delayed beyond the parent-set bedtime ${ }^{5)}$ and for older children for whom extinction may not be appropriate. The idea behind this method is to create pressure to sleep by delaying the bedtime to a time that fits the child's natural tendency. ${ }^{29)}$ Positive routines involve the parents developing enjoyable bedtime activities. When the association between positive bedtime events and falling asleep is established, the bedtime is moved earlier by $15 \mathrm{~min}$. utes over successive nights until the bedtime goal is reached. ${ }^{17)} \mathrm{A}$ modified version of this method, faded bedtime with response cost, involves taking the child out of bed (response cost) when unable to fall asleep to decrease the negative association between being in bed and being awake. ${ }^{17,26)}$ After a predetermined time (usually about 30 minutes) of a nonarousing activity, the child returns to bed. This procedure is repeated until the child falls asleep. It is essential to maintain a consistent morning wake-up time. ${ }^{17)}$

\section{4) Scheduled awakenings}

Scheduled awakenings involve parents awakening and typically consoling their child (e.g., feeding, rocking, soothing) approximately 15-30 minutes before a typical spontaneous awakening. ${ }^{1728)}$ Scheduled awakenings are then faded out by systematic increasing of the time span between awakenings. Scheduled awakening is a treatment option for frequent nighttime awakenings when the child awakens at relatively fixed hours that appears to increase the duration of consolidated sleep. ${ }^{16}$ )

\section{5) Sleep hygiene}

"Sleep hygiene" refers to modifiable environmental and behavioral factors that promote good sleep. ${ }^{8)}$ Parent education on sleep and/or sleep hygiene is important in all behavioral interventions. A systemic review of the evidence behind pediatric sleep practice recommendations showed a strong evidence base for: (1) consistent bedtime routines, (2) no electronics in the bedroom or before bed, and (3) independence when falling asleep, i.e., the child is not dependent on parental presence to fall asleep at bedtime or after night waking. ${ }^{8,33)}$ The avoidance of caffeine (e.g., chocolate, soft drinks, etc.) in the late afternoon is another recommendation with moderate-strength evidence. ${ }^{8,33)}$

\section{6) Parent education}

There is a gap between a baby's actual sleep and the parental expectations about it. Therefore, parents should first recognize whether their child actually has a sleep problem. ${ }^{26}$ Unfortunately, parents often do not discuss their child's sleep issues with health care professionals because they lack knowledge about pediatric sleep and sleep problems. ${ }^{26,34)}$ Furthermore, health care providers often do not ask about the child's sleep due to a lack of training and knowledge. ${ }^{26,34)}$

It is recommended that parents put the babies to bed "drowsy but awake" to help them learn to initiate sleep independently 
and return to sleep without requiring intervention, the so-called self-soothing skills. ${ }^{17)}$ Consistent bedtime routines and sleep schedules are critical targets for parent education programs.

\section{Pharmacologic therapy}

Behavioral interventions are the first choice for the treatment of pediatric insomnia, and medication should be used in combination with nonpharmacological strategies, especially in typically developing young children. ${ }^{35)}$ Specific pharmacological agents are classified into antihistamines, melatonin, melatonin receptor agonists, benzodiazepines, nonbenzodiazepines, alphaagonists, and atypical antidepressants. Melatonin is mainly used in patients with attention-deficit/hyperactivity disorder (ADHD), autism spectrum disorders (ASD), and other neurodevelopmental disabilities. Studies have shown that melatonin reduces the sleep-onset delay and increases total sleep duration in these patients (reported doses for melatonin: 3-6 mg per night for ADHD group, 1-3 mg for children with ASD, and a dose range of $0.5-10 \mathrm{mg}$ for children with special needs). ${ }^{36-38)}$

\section{Outcomes and long-term effects of behavioral interventions}

Many parents worry about the harmful effects of ignoring their child's nighttime crying on their emotion and development. It is important to emphasize to parents that most behavioral sleep interventions involve some level of infant protest and that no published studies show such adverse effects. ${ }^{29)}$ Several randomized studies showed that behavioral sleep treatments do not lead to later emotional and behavioral problems or negative impacts on the parent-child relationship ${ }^{25)}$ at 5 -year followup. ${ }^{39)}$ To the contrary, a longitudinal study showed that, in the condition of no sleep intervention, sleep problems (short sleep duration [ $\leq 10$ hours], and frequent nocturnal awakenings [ $\geq 3$ times]) at 18 months predicted a later incidence of emotional and behavioral problems at 5 years. ${ }^{40)}$

\section{Conclusions}

Behavioral treatments for young children result in significant improvements in sleep-onset latency and night waking without later causing emotional problems or negatively affecting the parent-child relationship. Parent education about a child's sleep and sleep hygiene is essential for treatment. Teaching parents within busy clinic time is challenging. Consultation with a clinical psychologist or trained nurse practitioner is needed to educate and support parents in this area.

\section{Conflicts of interest}

No potential conflicts of interest relevant to this article are reported.

\section{References}

1. Weinraub M, Bender RH, Friedman SL, Susman EJ, Knoke B, Bradley R, et al. Patterns of developmental change in infants' nighttime sleep awaken. ings from 6 through 36 months of age. Dev Psychol 2012;48:1511-28.

2. Meltzer LJ, Mindell JA. Systematic review and meta-analysis of behavioral interventions for pediatric insomnia. J Pediatr Psychol 2014;39:932-48.

3. Iglowstein I, Jenni OG, Molinari L, Largo RH. Sleep duration from in fancy to adolescence: reference values and generational trends. Pediatrics 2003;111:302-7.

4. Paruthi S, Brooks LJ, D'Ambrosio C, Hall WA, Kotagal S, Lloyd RM, et al. Recommended amount of sleep for pediatric populations: a consensus statement of the american academy of sleep medicine. J Clin Sleep Med 2016;12:785-6.

5. Owens JA, Moore M. Insomnia in infants and young children. Pediatr Ann 2017;46:e321-6.

6. Chaput JP, Gray CE, Poitras VJ, Carson V, Gruber R, Birken CS, et al. Systematic review of the relationships between sleep duration and health indicators in the early years (0-4 years). BMC Public Health 2017;17:855.

7. Meltzer LJ, Mindell JA. Relationship between child sleep disturbances and maternal sleep, mood, and parenting stress: a pilot study. J Fam Psychol 2007;21:67.

8. Hill CM, Everitt H. Assessment and initial management of suspected behavioural insomnia in pre-adolescent children. BMJ 2018;363:k3797.

9. Sateia MJ. International classification of sleep disorders-third edition: highlights and modifications. Chest 2014;146:1387-94.

10. American Academy of Sleep Medicine. International classification of sleep disorders (ICSD-3). 3rd ed. Darien (IL): American Academy of Sleep Medicine, 2014.

11. Mindell JA, Owens JA. Bedtime problems in young children. A clinical guide to pediatric sleep: diagnosis and management of sleep problems. 3rd ed. Philadelphia (PA): Wolters Kluwer Health/Lippincott Williams \& Wilkins, 2015:75-82.

12. Bruni O, Sette S, Angriman M, Baumgartner E, Selvaggini L, Belli C, et al. Clinically oriented subtyping of chronic insomnia of childhood. J Pediatr 2018;196:194-200.e1.

13. Meltzer LJ, Johnson C, Crosette J, Ramos M, Mindell JA. Prevalence of diagnosed sleep disorders in pediatric primary care practices. Pediatrics 2010;125:e1410-8.

14. Souders MC, Mason TB, Valladares O, Bucan M, Levy SE, Mandell DS, et al. Sleep behaviors and sleep quality in children with autism spectrum disorders. Sleep 2009;32:1566-78.

15. Calhoun SL, Fernandez-Mendoza J, Vgontzas AN, Liao D, Bixler EO. Prevalence of insomnia symptoms in a general population sample of young children and preadolescents: gender effects. Sleep Med 2014;15: 91-5.

16. Kim DS, Lee CL, Ahn YM. Sleep problems in children and adolescents at pediatric clinics. Korean J Pediatr 2017;60:158-65.

17. Mindell JA, Kuhn B, Lewin DS, Meltzer LJ, Sadeh A. Behavioral treatment of bedtime problems and night wakings in infants and young children. Sleep 2006;29:1263-76.

18. Mindell JA, Sadeh A, Kwon R, Goh DY. Cross-cultural differences in the sleep of preschool children. Sleep Med 2013;14:1283-9.

19. Ahn Y, Williamson AA, Seo HJ, Sadeh A, Mindell JA. Sleep patterns among South Korean infants and toddlers: global comparison. J Korean Med Sci 2016;31:261-9.

20. Owens JA, Dalzell V. Use of the 'BEARS' sleep screening tool in a pediatric residents' continuity clinic: a pilot study. Sleep Med 2005;6:63-9.

21. Sadeh A. A brief screening questionnaire for infant sleep problems: validation and findings for an Internet sample. Pediatrics 2004;113:e570-7.

22. Camerota M, Tully KP, Grimes M, Gueron-Sela N, Propper CB. Assessment of infant sleep: how well do multiple methods compare? Sleep 2018;41:zsy146.

23. Tikotzky L, Volkovich E. Infant nocturnal wakefulness: a longitudinal study comparing three sleep assessment methods. Sleep 2019;42. https:// doi.org/10.1093/sleep/zsy191. 
24. Werner H, Hunkeler P, Benz C, Molinari L, Guyer C, Häfliger F, et al. The Zurich 3-step concept for the management of behavioral sleep disorders in children: a before-and-after study. J Clin Sleep Med 2015;11:241-9.

25. Gradisar M, Jackson K, Spurrier NJ, Gibson J, Whitham J, Williams AS, et al. Behavioral interventions for infant sleep problems: a randomized controlled trial. Pediatrics 2016;137:e20151486.

26. Vriend J, Corkum P. Clinical management of behavioral insomnia of childhood. Psychol Res Behav Manag 2011;4:69-79.

27. Kahn M, Juda-Hanael M, Livne-Karp E, Tikotzky L, Anders TF, Sadeh A. Behavioral interventions for pediatric insomnia: one treatment may not fit all. Sleep 2020;43:zsz268.

28. Rickert VI, Johnson CM. Reducing nocturnal awakening and crying episodes in infants and young children: a comparison between scheduled awakenings and systematic ignoring. Pediatrics 1988;81:203-12.

29. Tikotzky L, Sadeh A. The role of cognitive-behavioral therapy in behavioral childhood insomnia. Sleep Med 2010;11:686-91.

30. Etherton H, Blunden S, Hauck Y. Discussion of extinction-based behavioral sleep interventions for young children and reasons why parents may find them difficult. J Clin Sleep Med 2016;12:1535-43.

31. Adams LA, Rickert VI. Reducing bedtime tantrums: comparison between positive routines and graduated extinction. Pediatrics 1989;84:756-61.

32. Piazza CC, Fisher WW. Bedtime fading in the treatment of pediatric insomnia. J Behav Ther Exp Psychiatry 1991;22:53-6.

33. Allen SL, Howlett MD, Coulombe JA, Corkum PV. ABCs of SLEEPING: a review of the evidence behind pediatric sleep practice recommendations. Sleep Med Rev 2016;29:1-14.

34. Schreck KA, Richdale AL. Knowledge of childhood sleep: a possible variable in under or misdiagnosis of childhood sleep problems. J Sleep Res 2011;20:589-97.

35. Kratochvil CJ, Owens JA. Pharmacotherapy of pediatric insomnia. J Am Acad Child Adolesc Psychiatry 2009;48:99-107.

36. Ferracioli-Oda E, Qawasmi A, Bloch MH. Meta-analysis: melatonin for the treatment of primary sleep disorders. PLoS One 2013;8:e63773.

37. Gringras P, Nir T, Breddy J, Frydman-Marom A, Findling RL. Efficacy and safety of pediatric prolonged-release melatonin for insomnia in children with autism spectrum disorder. J Am Acad Child Adolesc Psychiatry 2017;56:948-57.e4.

38. Esposito S, Laino D, D’Alonzo R, Mencarelli A, Di Genova L, Fattorusso A, et al. Pediatric sleep disturbances and treatment with melatonin. J Transl Med 2019;17:77.

39. Price AM, Wake M, Ukoumunne OC, Hiscock H. Five-year follow-up of harms and benefits of behavioral infant sleep intervention: randomized trial. Pediatrics 2012;130:643-51.
40. Sivertsen B, Harvey AG, Reichborn-Kjennerud T, Torgersen L, Ystrom E, Hysing M. Later emotional and behavioral problems associated with sleep problems in toddlers: a longitudinal study. JAMA Pediatr 2015;169:57582.

41. Richman N, Stevenson JE, Graham PJ. Prevalence of behaviour problems in 3-year-old children: an epidemiological study in a London borough. J Child Psychol Psychiatry 1975;16:277-87.

42. Richman N, Graham PJ. A behavioural screening questionnaire for use with three-year-old children. Preliminary findings. J Child Psychol Psychiatry 1971;12:5-33.

43. Camhi SL, Morgan WJ, Pernisco N, Quan SF. Factors affecting sleep disturbances in children and adolescents. Sleep Med 2000;1:117-23.

44. Archbold KH, Pituch KJ, Panahi P, Chervin RD. Symptoms of sleep disturbances among children at two general pediatric clinics. J Pediatr 2002;140:97-102.

45. Chervin RD, Hedger K, Dillon JE, Pituch KJ. Pediatric sleep questionnaire (PSQ): validity and reliability of scales for sleep-disordered breathing, snoring, sleepiness, and behavioral problems. Sleep Med 2000;1:21-32.

46. Jiang F, Shen X, Yan C, Wu S, Jin X, Dyken M, et al. Epidemiological study of sleep characteristics in Chinese children 1-23 months of age. Pediatr Int 2007;49:811-6.

47. Mindell JA, Meltzer LJ, Carskadon MA, Chervin RD. Developmental aspects of sleep hygiene: findings from the 2004 National Sleep Foundation Sleep in America Poll. Sleep Med 2009;10:771-9.

48. Zhang J, Li AM, Kong AP, Lai KY, Tang NL, Wing YK. A communitybased study of insomnia in Hong Kong Chinese children: prevalence, risk factors and familial aggregation. Sleep Med 2009;10:1040-6.

49. Mindell JA, Sadeh A, Wiegand B, How TH, Goh DY. Cross-cultural differences in infant and toddler sleep. Sleep Med 2010;11:274-80.

50. Simola P, Laitalainen E, Liukkonen K, Virkkula P, Kirjavainen T, Pitkaranta A, et al. Sleep disturbances in a community sample from preschool to school age. Child Care Health Dev 2012;38:572-80.

51. Bruni O, Ottaviano S, Guidetti V, Romoli M, Innocenzi M, Cortesi F, et al. The Sleep Disturbance Scale for Children (SDSC). Construction and validation of an instrument to evaluate sleep disturbances in childhood and adolescence. J Sleep Res 1996;5:251-61.

How to cite this article: Kang EK, Kim SS. Behavioral insomnia in infants and young children. Clin Exp Pediatr 2021;64:111-6. https://doi.org/10.3345/cep.2020.00052 